\section{Ultrasound guidance and success rates of axillary brachial plexus block - II}

To the Editor:

We read with great interest Chan et al.'s ${ }^{1}$ study comparing ultrasound with nerve stimulation for axillary plexus block. Despite increased use of ultrasound in regional anesthesia, randomized trials comparing this approach with modern nerve stimulation techniques are relatively few. ${ }^{2}$ However, in our opinion this study missed an opportunity to fully investigate the role of ultrasound for axillary block.

Unfortunately, ultrasound in Chan et al.'s ${ }^{1}$ study did not utilize current nerve stimulation modalities for axillary block. ${ }^{3-5}$ The authors reported a success rate of $62.9 \%$ for median, ulnar and radial sensory block, which is relatively low compared with currently published data (> 92\% success rates) and our clinical experience. ${ }^{6}$ Despite this discrepancy, the authors conclude that ultrasound "significantly improves the success rate." We also note a slight error in Table II for "Successful surgical anesthesia without supplementation". ${ }^{1}$ For the ultrasound group, we believe the reported percentage should have been $92.2 \%$ rather than $95 \%$ (59 of 64 , not 62 patients). This changes the $P$ value (but not overall significance) from 0.07 to 0.26 .

Studies demonstrating success rates of $91-97 \%$ have accepted a distal response as the only endpoint for radial nerve stimulation. Coventry et al. ${ }^{3}$ performed triple stimulation axillary block $(25 \mathrm{~mL}$ lidocaine $1.5 \%$ with epinephrine), reporting $100 \%$ sensory blockade of median, ulnar and radial nerves to pinprick at 30 min. Sia et al. ${ }^{4}$ performed triple stimulation axillary block (36 mL lidocaine $2 \%$ and bupivacaine $0.5 \%$ ). $\mathrm{He}$ reported success rates of $93 \%$ for median and ulnar sensory block (cold at $30 \mathrm{~min}$ ) and 95\% for the radial nerve. Chan et al. ${ }^{1}$ accepted triceps rather than distal responses as an appropriate endpoint in $85 \%$ of patients despite $\mathrm{Sia}^{5}$ demonstrating a significant difference in sensory radial nerve blockade when comparing a proximal (triceps) with a distal (wrist/fingers) endpoint ( $81 \%$ vs $95 \%)$. The accompanying editorial alluded to the low success rate, but the findings were explained by "rigid definitive endpoints of complete pinprick", and despite stating that the proximal response "may have been detrimental", concluded "clearly, ultrasound visibility of the nerve will enhance success". ${ }^{2}$

If we are to use ultrasound guidance for axillary block in everyday practice, we must first compare the technique with the presently-accepted standard of seeking distal radial responses in triple stimulation axillary blocks.

Stephen Mannion MB MRCPI FCARCSI MD

Xavier Capdevila MD PhD

Hôpital Lapeyronie, Montpellier, France E-mail: mannionstephen@hotmail.com Accepted for publication April 16, 2007.

\section{References}

l Chan VW, Perlas A, McCartney CJ, Brull R, Xu D, Abbas $S$. Ultrasound guidance improves success rate of axillary brachial plexus block. Can J Anesth 2007; 54: 176-82.

2 Tsui B. Ultrasound-guidance and nerve stimulation: implications for the future practice of regional anesthesia (Editorial). Can J Anesth 2007; 54: 165-70.

3 Coventry DM, Barker KF, Thomson M. Comparison of two neurostimulation techniques for axillary brachial plexus blockade. Br J Anaesth 2001; 86: 80-3.

4 Sia S, Lepri A, Magherini M, Doni L, Di Marco P, Gritti $G$. A comparison of proximal and distal radial nerve motor responses in axillary block using triple stimulation. Reg Anesth Pain Med 2005; 30: 458-63.

5 Sia S. A comparison of injection at the ulnar and the radial nerve in axillary block using triple stimulation. Reg Anesth Pain Med 2006; 31: 514-8.

6 Handoll H, Koscielniak-Nielsen ZJ. Single, double or multiple injection techniques for axillary brachial plexus block for hand, wrist or forearm surgery, Cochrane Database Syst Rev 2006; 25: CD003842.

\section{Reply:}

We sincerely thank Drs. Mannion and Capdevila and also Drs. Aguirre, Blumenthal and Borgeat for their interest in our article, and take this opportunity to clarify some of the issues which they raised.

The major concern is related to the apparently low success rate of axillary block associated with triple endpoint nerve stimulation (62.9\% in group NS) as compared to $80.7 \%$ and $82.8 \%$ with ultrasound + nerve stimulation (group USNS) and ultrasound alone (group US), respectively. ${ }^{1}$ As stated in the manuscript, our definition of block success was complete sensory anesthesia to pinprick in all three target nerves (radial, ulnar and median) at $30 \mathrm{~min}$. This strict definition is more rigorous than commonly-used endpoints e.g., successful surgical anesthesia or loss of cold sensation.

As stated by Aguirre et al., Stan ${ }^{2}$ reported "successful surgical anesthesia” in $88.8 \%$ of patients receiving trans-arterial axillary blocks. When this definition is 
applied, we observe comparable rates of block success (85.5\%, 92\% and 95\% in groups NS, USNS and US respectively) in the present study. ${ }^{1}$ Our results are clearly not different from other studies using similar endpoints. Most importantly, the same rigorous definition of block success was applied across all study groups, thus not biasing against group NS.

We disagree with the notion that the accepted endpoint of a proximal radial motor response had a major impact on our study results. Sia ${ }^{3}$ compared distal and proximal radial motor response during axillary block and concluded that seeking a distal motor response could enbance blockade of "the sensory distal branches of the radial nerve" (distal 95\% vs proximal 81\%) only. However, neither the overall block success rate (blockade of median, ulnar, radial and musculocutaneous nerves) nor the onset time were improved by intentionally seeking a distal radial motor response. ${ }^{3}$ Furthermore, it is interesting to point out that in the present study, a proximal radial motor response was observed in a majority of patients in both groups NS and USNS, yet the overall block success rate was significantly higher in group USNS (80.7\% vs 62.9\% in group NS).

We acknowledge an error in Table II. The count of successful surgical anesthesia without supplementation should read 61/64 instead of 59/62 in group US with no change in the percentage (95\%) or the P value (0.07).

In our opinion, experts who can consistently achieve block success of $90 \%$ or higher with traditional paresthesiae or nerve stimulation techniques may not need ultrasound guidance to enbance block success rates. However, assessment of local anesthetic spread, early detection of an intraneural and intravascular injection, and prevention of a pleural puncture are some of the distinct features unique to ultrasound which surpass traditional nerve block techniques. We believe that before we can conclusively address clinical outcomes and develop evidence-based practice of ultrasound guided regional techniques, large scale randomized controlled trials are needed, and our recently published study is one step in the right direction.

Anahi Perlas MD FRCPC

Vincent W.S. Chan MD FRCPC

Richard Brull MD FRCPC

Toronto Western Hospital, University Health Network, University of Toronto, Toronto, Canada E-mail: anahi.perlas@uhn.on.ca

\section{References}

I Chan VW, Perlas A, McCartney CJ, Brull R, Xu D, Abbas $S$. Ultrasound guidance improves success rate of axillary brachial plexus block. Can J Anesth 2007; 54:
176-82.

2 Stan TC, Krantz MA, Solomon DL, Poulos JG, Chaouki $K$. The incidence of neurovascular complications following axillary brachial plexus block using a transarterial approach. A prospective study of 1,000 consecutive patients. Reg Anesth 1995; 20: 486-92.

3 Sia S, Lepri A, Magherini M, Doni L, Di Marco P, Gritti $G$. A comparison of proximal and distal radial nerve motor responses in axillary block using triple stimulation. Reg Anesth Pain Med 2005; 30: 458-63.

\section{Electrical stimulation: an important force behind the growth of regional anesthesia}

To the Editor:

Having had the privilege to participate in clinical care and research of patients enjoying the well-described benefits of peripheral nerve block analgesia within the past 20 years, we read with interest the recent editorial by Dr. Ban Tsui in the Journal. ${ }^{1}$ In his editorial, Dr. Tsui described the evolution of ultrasound imaging, and its role in increasing the availability of peripheral regional techniques to patients.

Overall, we found the editorial to be very wellwritten and balanced. Its content raised important questions, and acknowledged that we do not have enough information yet to answer most of those questions. The author's acknowledgment regarding personal experiences with earlier-version ultrasound software was appropriate, and certainly forthcoming technological improvements with imaging should be valuable. We generally agree with the editorial's overall message.

Dr. Tsui, however, stated that in contrast with the clinical popularity evolving with ultrasound imaging in peripheral nerve blocks, "Not surprisingly, the introduction of (electrical stimulation) failed to result in a renewed interest in regional anesthesia." We respectfully disagree with this statement based on our collective research and experience with electrical stimulation, at least in the context of when neurostimulation was made available commercially in a sufficiently "user-friendly" format. We will illustrate our point with a brief example.

A prominent vendor of peripheral nerve stimulation equipment and supplies shared sales data with us (1998-present). This vendor has appropriately requested anonymity for this report. The annual percentage sales growth (when compared with the previous year, not the baseline year) is shown in the Figure. Data are also provided regarding the number 